\title{
Understanding hospital referral rates: a user's guide
}

\author{
M O Roland, J Bartholomew, D C Morrell, A McDermott, E Paul
}

\begin{abstract}
Detailed referral information from one practice was used to investigate the effect of calculating referral rates in several different ways. Referral rates for individual general practitioners should be related to the number of consultations carried out and not to the number of registered patients; for whole practices list size may be used as the denominator. Most doctors will not need to control for age and sex of patients when comparing referral rates but may need to control for case mix when comparing referral rates to individual specialties. In addition, a method is described for distinguishing systematic variation between the referral rates of individual doctors from the random variation that may arise from data based on fairly small numbers of referrals. The method indicates whether systematic variation is greater than would be expected by chance, and it can be extended to indicate whether variability in referral rates is greater in one specialty than another. Because of random variation with time a year's data may not be sufficient to allow reliable interpretation of referral rates to individual specialties, except for the largest.
\end{abstract}

At present there is no known relation between high or low referral rates and quality of care. Nevertheless, if doctors are to interpret their own rates of referral they need those rates to be reliable and valid. Use of the 10 guidelines described in this paper will help to prevent unwarranted conclusions being drawn from information on general practitioners' rates of referral to hospital.

\section{Introduction}

It has been known for many years that the rate at which general practitioners refer patients to hospital varies not just within different regions of the country but among practices within defined districts. ${ }^{2}$ Considerable differences have also been identified between doctors within a single practice. ${ }^{34}$ Some careful analyses have allowed for age of doctors, list sizes, postgraduate qualifications, practice arrangements, and standardisation for age and sex and case mix, but none of these studies have effectively explained these differences in referral rates.

High rates of referral to hospital may reflect excessive use of expensive resources, but it may be that lower referral rates reflect the general practitioner's lack of sensitivity to the needs of patients for specialist care. Before either of these hypotheses can be tested it is essential to determine the validity of a number of ways of presenting referral rates. The aim of this paper is to provide guidelines that relate to some of the problems in measuring and presenting information on referrals. In particular, we investigated the problem of which denominator to use; the value of standardising for age, sex, and case mix; and the problem of distinguishing random from systematic variation. We used detailed referral data collected from one practice to illustrate these points.

\section{Method}

The study took place in the Lambeth Road group practice, London, which provides primary care for about 10000 people. This is an inner city academic practice in which all the principals are salaried employees; in addition to providing patient care they carry out educational programmes and research. Although this practice is atypical in some respects, it offers a setting where the detailed and accurate information required for this type of study can be collected. The practice employs two trainees, and during the period of the study one principal, who had worked in the practice for 15 years, resigned. The study was therefore based on the work of six principals, two trainees, and a long term locum. One trainee was present in the practice throughout the study and one for two thirds of the time. The practice reflects the sort of instability of staffing that often affects a large group practice.

The data were collected in two ways. Firstly, for a period of six months the doctor was provided at each consulting session with an encounter sheet listing the patients booked to see him or her in that surgery. Spaces were left for patients who attended without an appointment. The patient's name, sex, and date of birth were written on the encounter sheet before the start of the surgery. After each consultation the doctor entered the main reason for the consultation and whether or not the patient was referred to hospital. If a referral was made the department to which the patient was referred was also noted. The doctor recorded the patient's main presenting problem in one of eight major clinical groups (medicine, surgery, gynaecology, psychiatry, locomotor disorders, ophthalmology, dermatology, and ear, nose, and throat), which matched up with the major departments to which referrals might be made. The coding system was tested before the main study and produced few problems apart from the coding of some presenting problems, for which definitions were agreed. Secondly, all letters typed and sent to hospitals requesting outpatient consultations over the period of the study were filed separately and subsequently counted. The referral rates recorded on the encounter sheet were expected to exceed the referrals recorded by hospital letters because it is well known that some referrals are urgent and instituted by handwritten letter and others negotiated over the telephone. This was in fact the case for most of the doctors, and the number of referrals recorded on the encounter sheet was used in all the analyses reported in this paper.

\section{Results}

Table I shows the number of referrals and consul-
Correspondence to:

BrMed f 1990;301:98-102 
tations carried out by each of the doctors. These data raise several questions.

\section{HOW SHOULD A REFERRAL RATE BE CALCULATED?}

A referral rate can be calculated using one of three denominators: the number of registered patients, the number of patients who consult, or the number of consultations.

\section{List size as denominator}

When one practice is compared with another the appropriate referral rates may be calculated using the practice list size as the denominator. In comparing individual doctors in a partnership the registered list size of the doctor or the average list size of the doctors in the practice may be used. Previous work has shown that individual list size is a poor reflection of workload and that average list size is a better denominator for comparing referral rates. ${ }^{6}$

In this study the percentage of patients who both consulted and were registered with individual doctors was calculated. Three of the doctors had no such patients as two were trainees and one a locum. Though they had no registered list, they carried out $35 \%$ of the consultations and made $30 \%$ of the referrals in the study period. Of consultations carried out with principals, $23-50 \%$ were with the patient's own registered doctor; thus the fact that a patient was registered with a particular doctor meant neither that he or she consulted with that doctor nor that he or she was referred by that doctor. Individual list size was therefore a meaningless denominator for comparing the referral patterns of the doctors in this study.

Average list size was not an ideal denominator as it did not reflect differences in workload among doctors within the practice. Partly because of varying outside commitments individual doctors had nearly a twofold range in the number of consultations they carried out during the study period (table I). This difference would have been obscured by using average list size as the denominator.

\section{Number of consultations or of patients consulting as denominator}

Both the number of consultations and the number of patients consulting have been used as denominators for calculating referral rates, notably in the national morbidity surveys. ${ }^{7}$ The number of consultations is the simplest measure of workload, but it may seem inflated in terms of new problems if one doctor in a practice is more likely to recall patients for follow up consultations than are the others. For this reason the number of different patients consulting has been proposed as a denominator.

TABLE I - Number of consultations and referrals and referral rate per 100 consultations for each doctor

\begin{tabular}{lccc}
\hline Doctor No & $\begin{array}{c}\text { No of consultations in } \\
\text { six months }\end{array}$ & $\begin{array}{c}\text { No of referrals recorded on } \\
\text { encounter sheets }\end{array}$ & $\begin{array}{c}\text { No of referrals/100 consultations } \\
(95 \% \text { confidence interval) }\end{array}$ \\
\hline 1 & 586 & 20 & $3 \cdot 4(1 \cdot 9$ to $4 \cdot 8)$ \\
2 & 1084 & 64 & $5 \cdot 9(4 \cdot 5$ to $7 \cdot 3)$ \\
3 & 1041 & 63 & $6 \cdot 0(4 \cdot 6$ to $7 \cdot 5)$ \\
4 & 1195 & 37 & $3 \cdot 1(2 \cdot 1$ to $4 \cdot 1)$ \\
5 & 1374 & 37 & $2 \cdot 6(1 \cdot 8$ to $3 \cdot 5)$ \\
6 & 1568 & 82 & $5 \cdot 2(4 \cdot 1$ to $6 \cdot 3)$ \\
7 & 855 & 40 & $4 \cdot 6(3 \cdot 3$ to $6 \cdot 1)$ \\
8 & 1353 & 68 & $5 \cdot 0(3 \cdot 9$ to $6 \cdot 2)$ \\
9 & 1497 & 143 & $9 \cdot 5(8 \cdot 1$ to $11 \cdot 0)$ \\
\hline
\end{tabular}

TABLE II - Age and sex specific referral rates per 1000 patents at risk (all referrals). Data from third national morbidity survey

\begin{tabular}{lrrrrrrrr}
\hline & All ages & \multicolumn{1}{c}{$0-4$} & $5-14$ & $15-24$ & $25-44$ & $45-64$ & $65-74$ & $\geqslant 75$ \\
\hline Men & $99 \cdot 2$ & $113 \cdot 5$ & $67 \cdot 4$ & $66 \cdot 7$ & $83 \cdot 3$ & $113 \cdot 2$ & $165 \cdot 2$ & $222 \cdot 9$ \\
Women & $134 \cdot 1$ & $85 \cdot 7$ & $60 \cdot 9$ & $142 \cdot 7$ & $160 \cdot 1$ & $129 \cdot 1$ & $142 \cdot 1$ & $193 \cdot 1$ \\
\hline Total & $117 \cdot 5$ & $100 \cdot 0$ & $64 \cdot 3$ & $106 \cdot 1$ & $123 \cdot 2$ & $121 \cdot 3$ & $152 \cdot 3$ & $203 \cdot 1$ \\
\hline
\end{tabular}

In this study the mean number of consultations per patient consulting in the six month study period was calculated for each of the doctors. This ranged from 1.22 for the doctor with the lowest recall rate to 1.77 for the doctor with the highest. The doctors with the higher recall rates were the older doctors who also had older patients with more chronic illness (see below). It was not therefore possible to tell whether the differences in recall rates were an effect of case mix or due to differences in the doctors' behaviour. Although this might suggest that it would be more appropriate to use the number of patients consulting as a denominator rather than the number of consultations, in practice the differences were small. The ratios of the referral rates of the highest and lowest referring doctors were $4 \cdot 3$ for numbers of patients consulting and $3 \cdot 6$ for the number of consultations. The rank order of referral rates by doctor was similar whichever denominator was chosen, and the effect of standardising for age and sex was similar for the two denominators.

Although the number of patients consulting might be a more appropriate denominator than the number of consultations, the differences between the two methods were not great in this study and probably would not justify the greatly increased difficulty in collecting data on the number of patients consulting, which requires consultations to be indexed on a computer by using a unique patient identifier.

\section{IS IT WORTH STANDARDISING FOR AGE AND SEX?}

Referral rates using each of the three denominators were standardised for age and sex both directly and indirectly. As broadly similar conclusions were reached using each method, the following results are presented for indirectly standardised rates using number of consultations as the denominator.

Standardisation had little effect on either the spread of referral rates or the rank order of doctors. The crude rates ranged from 26.9 to 95.5 referrals per 1000 consultations, whereas the standardised rates ranged from 25.9 to 82.3 . The amount of variation between doctors was reduced by about $10 \%$ by standardisation, with the ratio between the highest and lowest referral rates reduced from 3.6 to 3.2 and the coefficient of variation of the referral rates reduced from 0.41 to $0 \cdot 37$. The effect of standardisation was therefore quite small when compared with the greater than threefold difference between doctors in their referral rates. The rank order of individual doctors' referral rates was identical for the crude and standardised rates.

This result is consistent with previous results. ${ }^{34}$ The reason that standardising for age and sex makes little difference can be seen by inspecting age and sex specific referral rates from the third national morbidity survey (table II). ${ }^{7}$ The range of referral rates is quite modest between the ages of 16 and 74 (from 106 to 152 referrals per 1000 patients at risk) compared with the size of differences in referral rates that is commonly observed between doctors, and it would seem unlikely that standardisation for age would have a significant effect except for practices or individual general practitioners who have an unusually high or low proportion of children or an unusual proportion of patients aged over 75. Likewise, the difference in referral rates of male and female patients is substantially less than the observed variation in general practitioners' referral rates and is unlikely to be important unless a doctor has an unusual proportion of female patients on his or her list, or unless it is necessary to explain quite small differences in doctors' referral rates.

If list size is being used as the denominator for calculating referral rates it is quite easy to standardise for age and sex, and there will be some benefit from doing so. If consultations are used as the denominator, however, standardisation is much more difficult, and it 
is probably not worth doing the extra data collection that would be required unless doctors are already collecting data on the age and sex of patients consulting.

\section{IS IT WORTH CONTROLLING FOR CASE MIX?}

There were clear differences between the types of patients seen by different doctors in this study. Female doctors were more likely to see gynaecological problems than were male doctors $(10.8 \%$ of consultations with female doctors compared with $4.9 \%$ of consultations with male doctors; $5 \cdot 9 \%$ difference, $95 \%$ confidence interval $4 \cdot 8 \%$ to $7 \cdot 0 \%$ ). There was also a clear relation between the age of the doctor and the age of the patient, with patients of the doctors who had been in the practice longest being, on average, 10 years older than patients of the trainees. These older patients were also more likely to consult repeatedly: $18.5 \%$ of patients of the three senior doctors consulted three times or more during the six month study period, compared with $9 \cdot 3 \%$ of the patients of any of the other doctors $(9 \cdot 2 \%$ difference, $7 \cdot 4 \%$ to $11 \%$ ).

We could calculate referral rates for individual specialties based on the number of consultations carried out in each specialty, but often doctors will not have information of this detail available. They may have been supplied with details of referrals broken down by diagnostic group but have available only the total number of consultations carried out by each doctor. We therefore wished to determine whether meaningful rates for individual diagnostic groups could be derived using the total number of consultations as the denominator. We calculated referral rates for individual diagnostic groups using the number of referrals in each group as the numerator and using both the total number of consultations across all groups and the number of consultations for that diagnostic group alone as denominators.

The two methods produced substantially different rank orders of referral rates for those diagnostic groups with appreciable differences in patterns of consultations between the doctors. For example, for gynaecology referrals, table III shows the rank order in referral rates calculated for the nine doctors by the two methods. The difference is particularly noticeable for doctor 3, who had the highest proportion of gynaecology consultations. She had the highest referral rate for gynaecology when the total number of consultations was used as the denominator but one of the lowest when the number of gynaecology consultations was used as the denominator. It follows that, where there are definite differences in the types of patient seen by different doctors, referral rates for individual diagnostic groups should be related to the number of consultations or of patients consulting for that diagnostic group and not just to the total number of consultations.

In terms of overall variability of referral rates, both methods of analysis identified the same diagnostic group-general medicine-as contributing more than other diagnostic groups to the overall variation in referral rates. The doctors in this practice might wish to direct their attention to this diagnostic group for clinical audit. These analyses do not, however, distinguish between variation due to systematic differences between doctors in referral rates and variation due to sampling error.

HOW MUCH OF THE APPARENT DIFFERENCE IN REFERRAL RATES WAS DUE TO CHANCE?

We found differences between doctors in their referral rates that were not consistent across diagnostic groups. One doctor had high referral rates in all diagnostic groups; others had high referral rates for some groups and low rates for others. Two doctors consistently had low referral rates.

Some of the variation in general practitioners' referral rates may be due to random variation in the presentation of patients requiring referral. This is a particular problem when dealing with small numbers of referrals - for example, a single specialty or diagnostic group over a limited period. ${ }^{\delta}$ We used the method of McPherson et al to distinguish the component of variance due to sampling error (random variation) from systematic variation between doctors (see appendix). ${ }^{9}$ The variation between doctors was compared by examining the ratio of the systematic to the random component of the variance using an $F$ test, in this case for each diagnostic group (table IV). Doctors whose expected number of referrals (that is, the number of consultations for that doctor multiplied by the referral rate in the practice) was less than five for a particular specialty were omitted from the analysis for that specialty. The expected number of referrals for psychiatry was less than five for most doctors, so psychiatric referrals were omitted from the analysis. For one specialty for which assumptions in the model led to the calculation of a small negative variance the variance was taken as equal to zero. General medicine was the only specialty for which the systematic variation in referrals was significant.

TABLE III - Effect of denominator (gynaecology consultations or total consultations) on rank order of gynaecology referral rates

\begin{tabular}{lccccc}
\hline Doctor No & $\begin{array}{c}\text { No of gynaecology } \\
\text { referrals }\end{array}$ & $\begin{array}{c}\text { No of gynaecology } \\
\text { consultations }\end{array}$ & $\begin{array}{c}\text { Referral rate/100 gynaecology } \\
\text { consultations (rank order) }\end{array}$ & $\begin{array}{c}\text { Total consultations } \\
\text { consultations (rank order) }\end{array}$ \\
\hline 1 & 2 & 22 & $9 \cdot 1(7)$ & 586 & $0 \cdot 34(7)$ \\
2 & 10 & 73 & $13 \cdot 7(4)$ & 1084 & $0 \cdot 92(5)$ \\
3 & 15 & 131 & $11 \cdot 5(6)$ & 1041 & $1 \cdot 44(1)$ \\
4 & 3 & 107 & $2 \cdot 8(9)$ & 1195 & $0 \cdot 25(8)$ \\
5 & 3 & 19 & $6 \cdot 1(8)$ & 1374 & $0 \cdot 22(9)$ \\
6 & 14 & 26 & $13 \cdot 9(3)$ & 855 & $0 \cdot 89(6)$ \\
7 & 1 & 150 & $12 \cdot 4(2)$ & 1353 & $1 \cdot 36(2)$ \\
8 & 18 & 67 & $20 \cdot 9(1)$ & 1497 & $0.33(3)$ \\
9 & 14 & & & & $0.93(4)$ \\
\hline
\end{tabular}

TABLE IV-Estimation of systematic and random components of variability in referral rates by diagnostic group

\begin{tabular}{|c|c|c|c|c|c|c|}
\hline & Total No of referrals & Total variance & $\begin{array}{c}\text { Systematic component of } \\
\text { variance between general } \\
\text { practitioners }\end{array}$ & $\begin{array}{l}\text { Random component of } \\
\text { variance }\end{array}$ & $\begin{array}{l}\text { Systematic component } \\
\text { divided by random } \\
\text { component }\end{array}$ & $\mathrm{p}$ Value \\
\hline General medicine & 123 & $0 \cdot 468$ & $0 \cdot 385$ & $0 \cdot 082$ & 4.675 & $0.022\left(F_{x, 8}\right)$ \\
\hline General surgery & 92 & $0 \cdot 109$ & 0.001 & $0 \cdot 108$ & 0.009 & $>0.5\left(F_{5,5}\right)$ \\
\hline Ear, nose, and throat & 46 & $0 \cdot 249$ & 0.075 & $0 \cdot 174$ & $0 \cdot 431$ & $>0.5\left(F_{5,5}\right)$ \\
\hline Ophthalmology & 47 & $0 \cdot 105$ & $\simeq 0$ & $0 \cdot 166$ & $\simeq 0$ & $>0 \cdot 5\left(F_{5,5}\right)$ \\
\hline Gynaecology & 83 & $0 \cdot 223$ & $0 \cdot 119$ & $0 \cdot 103$ & $1 \cdot 155$ & $0.433\left(F_{6,6}\right)$ \\
\hline Locomotor disorders & 57 & $0 \cdot 161$ & 0.008 & $0 \cdot 153$ & $0 \cdot 052$ & $>0 \cdot 5\left(F_{6,6}\right)$ \\
\hline Dermatology & 54 & 0.191 & 0.035 & $0 \cdot 156$ & $0 \cdot 226$ & $>0.5\left(F_{6,6}\right)$ \\
\hline All specialties & 554 & $0 \cdot 139$ & $0 \cdot 121$ & 0.018 & $6 \cdot 72$ & $0 \cdot 007\left(F_{8,8}\right)$ \\
\hline
\end{tabular}




\begin{tabular}{lccc}
\hline Doctor No & $\begin{array}{c}\text { Actual No of general medical } \\
\text { referrals }\end{array}$ & $\begin{array}{c}\text { Expected No of general } \\
\text { medical referrals }\end{array}$ & $\begin{array}{c}\text { Actual minus expected } \\
\text { number of referrals }\end{array}$ \\
\hline 1 & 2 & $6 \cdot 4$ & $-4 \cdot 4$ \\
2 & 14 & $13 \cdot 5$ & $0 \cdot 5$ \\
3 & 10 & $10 \cdot 1$ & $-0 \cdot 1$ \\
4 & 10 & $12 \cdot 9$ & $-2 \cdot 9$ \\
5 & 11 & $14 \cdot 9$ & $-3 \cdot 9$ \\
6 & 10 & $19 \cdot 9$ & $-9 \cdot 9$ \\
7 & 2 & $9 \cdot 7$ & $-7 \cdot 7$ \\
8 & 11 & $15 \cdot 6$ & $32 \cdot 8$ \\
9 & 53 & $20 \cdot 2$ & \\
\hline
\end{tabular}

$\star$ Practice medical referral rate $\times$ individual doctor's general medical consultations.

In the case of general medicine the number of referrals made by each doctor was compared with an expected number of referrals calculated by applying the practice referral rate for medicine to the number of general medical consultations undertaken by each individual doctor. The results (table V) suggest that if these doctors wished to examine their referral patterns attention might reasonably be directed towards doctor 9 , who made over 30 more medical referrals than expected, and doctor 6, who referred 10 fewer than expected.

In addition, the data in table IV can be used to examine the relative variability between specialties. In this case the systematic component of the variance of two diagnostic groups can be compared by examining their ratio using an $F$ test. Though general medicine stands out as having the greatest variability, there is also considerable variability between other specialties - for example, there is significantly more variability in gynaecology than dermatology $\left(F_{6,6}=0 \cdot 119 / 0 \cdot 035=\right.$ $5.69 ; \mathrm{p}<0.005)$. The analysis presented here is based on referrals over a six month period by doctors in one practice. Greater variability in referral rates both within and between specialties would be expected in a sample that consisted of doctors from various practices.

\section{Discussion}

General practitioners are now required to supply family practitioner committees with details of their hospital referrals by specialty. Family practitioner committees will analyse the returns and identify abnormally high or low referral rates. ${ }^{10}$ We have investigated how referral rates may be analysed and presented in a meaningful form using detailed data from one south London group practice in which patients are encouraged to consult with one doctor, not necessarily the one with whom they are registered; in which trainees are employed; and in which a change in one principal took place during the study period. We believe that these circumstances are not unusual and that they highlight certain problems in interpreting referral rates. On the basis of our work we suggest that family practitioner committees and general practitioners might use the following ten guidelines to help them interpret data on referrals.

(1) In the case of singlehanded doctors, referral rates may meaningfully be calculated by dividing the number of referrals by the number of patients registered with that doctor.

(2) In the case of group practices, a practice referral rate may meaningfully be calculated by dividing the total number of referrals by the total list size.

(3) Practice based referral rates are unlikely to be a useful basis for the "self audit and voluntary peer review" that the government proposes or for "discussions between the individual GP and the FPC to help reveal why the referral decisions were made." these purposes general practitioners will need to know their individual referral rates.
(4) Individual list size should not be used as a denominator for calculating an individual referral rate as it takes no account of major differences in workload, which may be unrelated to the number of patients registered with a doctor.

(5) Average practice list size may be preferable to individual list size as a denominator for calculating referral rates, but it still obscures substantial differences in workload and may distort referral rates by a factor of two to three.

(6) The number of consultations carried out by a doctor is the most satisfactory denominator for calculating a referral rate. Using the number of patients consulting has theoretical advantages over the number of consultations, but in practice the differences are not great enough to justify the increased complexity of data collection required to record the number of patients consulting.

(7) Bearing in mind the wide differences that are known to occur in general practitioners' referral rates and the comparatively small effect of standardisation of referral rates for age and sex, such standardisation is unlikely to have an important effect on the interpretation of referral rates except where a doctor or practice has an exceptionally high or low proportion of children or patients aged over 75. Standardisation is fairly simple if list size is used as the denominator for calculating referral rates, and may be of some benefit. Standardisation is much more difficult if consultations are used as the denominator, and it is probably not worth doing the extra data collection that is required.

(8) Standardisation for case mix is unlikely to be important when comparing overall practice referral rates. Individual doctors within practices may have special interests or areas of specialisation, and then interpretation of a high or low referral rate to one specialty may require knowing the doctor's workload in that area of clinical practice.

(9) Because of random variations with time a year's data may be insufficient to allow reliable interpretation of referral rates to individual specialties, except for the largest. For smaller specialties several years' data collection may be needed for comparing referral rates of individual general practitioners.

(10) The method described in the appendix can be used to distinguish random from systematic variation in referral rates. Applying this method could prevent health authorities and family practitioner committees from drawing attention to apparent differences in referral rates that are not related to doctors' referral behaviour. The method can also be used to identify areas of clinical practice that show great variability and might merit further investigation.

Up to now no relation has been found between quality of care and high or low referral rates. We do not know whether the patients of doctors with high referral rates use unnecessary resources and are exposed to unnecessary risk by being referred to hospital. Likewise, it is unclear whether the patients of doctors with low referral rates are being deprived of treatment from which they could benefit. Examining individual referral decisions, particularly in collaboration with local consultants, ${ }^{11}$ may benefit patient care. Examining referral statistics on their own is fraught with hazard. We have outlined some steps that should be used to ensure that a referral rate at least reflects the true likelihood that a doctor will refer a patient to hospital. Beyond that bald statistic, meaningful interpretation requires review of referral practice by the general practitioner, perhaps in association with his or her peers.

We thank Mary Evans and Lauralee White for help in handling the data in this study and the doctors of the Lambeth Road group practice. The study was supported by a grant from the Department of Health. 
Appendix

If there were no systematic differences between doctors in their referral rates there would still be sampling error (random variation) in the referral rates measured from a sample of doctors. The amount of this random variation in a specialty will depend on both the overall number of patients who consult for that specialty and the average referral rate for the specialty.

If there are systematic differences between doctors' referral rates the total variation measured between doctors will have a component that is due to systematic differences between doctors and a component that is due to sampling error (random variation, which will depend on the number of patients seen and the general level of referrals).

Let $\quad \mathrm{O}_{i}=$ observed number of referrals for doctor $\mathrm{j}$

and $\quad E_{j}=$ expected number of referrals for doctor $j$ if he referred at the same rate as the rest of the practice $=$ number of consultations for doctor $\mathrm{j} \times$ practice referral rate.

Assume $O_{j}=\lambda_{j} E_{j}$ where $\lambda_{j}$ is a factor associated with doctor $j$ that may vary from doctor to doctor. If $\lambda_{j}$ has the value 1 for all doctors then there is no systematic difference in their referral rates. If $\lambda_{j}$ varies with non-zero variance then there are systematic differences in the rates at which doctors refer.

Consider $\lambda_{i}$ as a random variable with an expected value of 1 and a variance of $\sigma^{2}$, and assume that the distribution of $\mathrm{O}_{j}$ is approximately Poisson, then

Variance $\left(\mathrm{O}_{i}\right)=\mathrm{E}_{\mathrm{i}}^{2} \sigma^{2}+\mathrm{E}_{\mathrm{i}}$

and we can estimate $\sigma^{2}$ by

Systematic variation $=$

$$
\hat{\sigma}^{2}=\frac{1}{k} \sum_{j=1}^{k}\left(\frac{\mathrm{O}_{i}-\mathrm{E}_{i}}{\mathrm{E}_{i}}\right)^{2}-\frac{1}{\mathrm{k}} \sum_{i=1}^{\mathrm{k}} \frac{1}{\mathrm{E}_{i}}
$$

$=$ Total variation - sampling error, where $\mathrm{k}$ is the number of doctors.

The ratio of the systematic component of variance within a specialty to the random component of variance can be compared with the $F$ distribution on $\mathrm{k}-1, \mathrm{k}-1$ degrees of freedom, to indicate whether the systematic variation between doctors' referral rates within a specialty is greater than would be expected by chance:

systematic variation

sampling error is distributed as $F_{\mathrm{k}-1, \mathrm{k}-1}$

Doctors whose expected numbers of referrals for a specialty are less than 5 should be omitted from the calculation of variation.

The $F$ test can also be used to compare the systematic components of variance for two specialties, to indicate whether variability in referral rates is greater in one specialty than in another:

systematic variation for specialty

systematic variation for specialty $i$ is distributed as $F_{\mathrm{k}-1, \mathrm{~m}-}$

where $k$ is the number of doctors included in the calculation of variation for specialty $j$ and $m$ is the number of doctors included in the calculation of variation for specialty $i$.

1 Scott R, Gilmore H. The Edinburgh hospitals. In: McLachlan G, ed. Problems and progress in medical care. London: Nuffield Provincial Hospitals Trust, 1966.

2 Wright HJ. General practice in south west England. London: Royal College of General Practitioners, 1968. (Reports from general practice No 8 .)

3 Morrell DC, Gage HG, Robinson NA. Referral to hospital by general practitioners. IR Coll Gen Prect 1971:21:77-85.

Cumins $R$, Jarman $B$, White $P$. Do general practitioners have different referral thresholds? I R Coll Gen Pract 1981;37:350-3.

5 Wilkin D, Smith A. Explaining variation in general practitioner referrals to hospital. Fam Prac 1987;4:160-9.

6 Armstrong D, Britten N, Grace J. Measuring general practitioners' referrals: patient workload and list size. $\mathcal{F} R$ Coll Gen Pract 1988;38:494-7.

7 Royal College of General Practitioners, Office of Population Censuses and Surveys, Department of Health and Social Security. Morbidity statistics from general practice 1981-1982. Third national study. London: HMSO, 1986.

8 Moore AT, Roland MO. How much variation in referral rates among general practitioners is due to chance? $\mathrm{Br}$ Med $\mathrm{f}$ 1989;298:500-2.

9 McPherson $\mathrm{K}$, Wennberg JE, Hovind $\mathrm{OB}$, Clifford $\mathrm{P}$. Small area variations in the use of common surgical procedures: an international comparison of New England, England and Norway. N Engl f Med 1982;307:1310-4

10 Health Departments of Great Britain Gencral practice in the National Health Service: the 1990 contract. London: Health Departments of Great Britain, Service: 1989.

11 Emmanuel J, Walter N. Referrals from general practice to hospital outpatient departments: a strategy for improvement. Br Med f 1989:299:722-4.

Accepted 9 May 1990

\section{ONE HUNDRED YEARS AGO}

It is not often the patient gets so hardly dealt with as in the German proverb which declares that "A physician is an angel when employed, but a devil when one must pay him." True, somebody said that "A disobedient patient makes an unfeeling physician," but what are these weak defences of our order in comparison with the terrible things with which the whole world of apophthegm pelts the unhappy doctor? The German aphorisms are particularly hard upon him. Here are some samples:- "Hussars pray for war and the doctors for fever," but in view of the new Notification of Diseases Act, they will probably cease to do that in this country. "A young physician should have three graveyards;" "A new doctor a new gravedigger;" "No physician is better than three," which is in opposition to the older maxim that "In a multitude of counsellors there is wisdom;" "New doctor new churchyard;" "Who has a physician has an executioner." All these sayings would seem to imply that however eminent the Germans may be in diagnosis, they are not uniformly successful in treatment. Spanish proverbs are grimly sarcastic at our expense "The earth hides as it takes the physician's mistakes;" "If you have a friend who is a physician, send him to the house of your enemy." They have a rhyme, too, which goes somewhat in this style:-

\section{And, doctor, do you really think \\ That asses' milk I ought to drink? \\ It cured yourself, I grant it true,}

But, then, 'twas mother's milk to you.

The French proverbs are not so polite towards us as they might be. For instance, "The doctor is often more to be feared than the disease;" and it was Voltaire who described a physician as "A man who pours drugs of which he knows little into a body of which he knows less." The Italians are not any better. "No good doctor ever takes physic;" "Physicians alone are permitted to murder with impunity." But the Greek comedian beats the record for attributing downright malice. "No physician takes pleasure in the health even of his best friend ",

There is a caustic humour about some Hebrew maxims which we should scarcely have expected. "Do not dwell in a city whose governor is a physician." "That city is in a bad case whose physician has the gout." Massinger said that "most physicians, as they grow greater in skill, grow less in religion;" but, however true that might have been in his time, it cannot be said now. We do not know who it was who uttered the sarcasm, "You need not doubt; you are no doctor." Nor can we say who the rude person was who elaborated the couplet,

$$
\begin{aligned}
& \text { With respect to the gout, } \\
& \text { The physician's but a lout. }
\end{aligned}
$$

Impertinent, certainly, but no fool was he.

Proverbs which deal with health apart from the doctors have more sense than some of those we have quoted. The Spaniard says, "He that sits with his back to a draught sits with his face to a coffin." "Of the malady a man fears he dies." Doubtless the treacherous nature of the climate of northern and central Spain is answerable for the following, "He that would be healthy must wear his winter clothes in summer."

The surgeon and his art do not appear in more than half a dozen of the "wise saws" of the world. "A good surgeon must have an eagle's eye, a lion's heart, and a lady's hand" is as respectful as it is true to the facts. Five of the six appear to be of English origin, the foreign one being of Italian: "Tender surgeons make foul wounds."

Medicine is the subject of some good maxims, such as the German one, "Dear physic always does good," and the Spanish, "What cures Sancho make Martha sick."

The Chinese have curious notions about zymotic diseases. "Starve the measles and nourish the small-pox" is scarcely intelligible on the germ theory, but it is quite as scientific as some of our own on the same lines. "One year a nurse and seven years the worse" is a little ambiguous. The Arabs say, "Among wonderful things is a sore-eyed person who is an oculist." "Diseases are the tax on our pleasures" is English in origin and application.

(British Medical fournal 1890;i:27) 